\title{
Calidad de vida relacionada con la salud de pacientes infectados por VIH medida por el Cuestionario de Salud SF-36
}

\author{
M. A. GARCÍA ORDÓÑEZ, J. J. MANSILLA FRANCISCO, E. NIETO ARAGÓN, M. \\ R. CERETO, F. SALAS SAMPER, M. VALLEJO DÍAZ, J. MARTÍNEZ GONZÁLEZ
}

\author{
Unidad de Enfermedades Infecciosas. Servicio de Medicina Interna. \\ Complejo Hospitalario Carlos Haya. Málaga.
}

\author{
HEALTH-RELATED OUALITY OF LIFE IN HIV-INFECTED \\ PATIENTS MEASURED BY HEALTH SURVEY SF-36
}

\section{RESUMEN}

Objetivos: Analizar el impacto de la infección por VIH sobre la Calidad de Vida Relacionada con la Salud (CVRS) en relación con variables clínicas e inmunológicas así como con la progresión y mortalidad de la enfermedad.

Pacientes y métodos: Estudio de una muestra de 300 pacientes infectados por VIH a los que se evaluó la CVRS mediante el cuestionario de salud SF-36 durante un periodo de 10 meses (mayo de 1997 a marzo de 1998). Como grupo control se incluyeron 300 individuos con hepatopatía crónica viral y potenciales factores de riesgo para la infección por VIH. Se calcularon los coeficientes alfa de Cronbach para la medición de la fiabilidad. Para relacionar la CVRS con la severidad de la enfermedad se utilizaron criterios clínicos (estadios de la CDC de Atlanta, diagnóstico previo de SIDA) y biológicos (recuento de linfocitos $\mathrm{CD}_{4}$ en sangre periférica). Se determinó la progresión y mortalidad de la enfermedad tras un seguimiento de 6 meses.

Resultados: Los pacientes con infección por VIH presentaron puntuaciones inferiores en las 8 dimensiones del SF- 36 respecto a los controles y a los valores poblacionales de referencia. Los valores del coeficiente alfa de Cronbach se situaron para todas las dimensiones por encima de 0,7 (recorri$\mathrm{do}=0,75-0,91$ ). Los pacientes en fases avanzadas de la enfermedad (con diagnóstico de SIDA y/o linfocitos $\mathrm{CD}<200 / \mathrm{mm}^{3}$ ) presentaron puntuaciones significativamente inferiores en todas las dimensiones salvo en salud mental. Treinta y siete $(14,2 \%)$ pacientes tuvieron nuevos eventos diagnósticos de SIDA y $21(8,1 \%)$ fallecieron. La inmunodepresión severa (OR: 4,3; IC 95\%: 1,6-11,8), presencia de SIDA previo (OR: 3,4; IC 95\%: 1,4$8,1)$ y las puntuaciones en las dimensiones del SF-36 función física (OR: 0,3; IC 95\%: 0,1-1,1) y dolor corporal (OR: 0,2; IC 95\%: 0,1-0,8) fueron factores predictivos de progresión y mortalidad de la enfermedad.

Conclusiones: El SF-36 como medida de la CVRS en individuos con infección por VIH muestra una alta consistencia interna, es capaz de discriminar a pacientes en estadios avanzados de la enfermedad y podría ayudar a predecir más adecuadamente el curso de la misma.

PALABRAS CLAVE: Calidad de vida. Cuestionarios. VIH. SIDA.

\section{ABSTRACT}

Objectives: The aim of the study was to known the influence of HIVinfection in healthrelated quality of life (HROL). We have used clinicoimmune variables and progressionmortality presented by the disease.

Patients and methods: From March 1997 through March 1998 a total of 300 HIV-infected patients were carried out the Health Survey $S F-36$. We included a control group (also 300 patients) who suffered chronic viral hepatitis and potencials risks for the HIV-infection. We cal culated the Cronbach s alpha coefficient to measure the reliability. We used clinical and biological datas (count of CD4 lymphocites in perip hery blood) to related the IIROL to the seerity of the disease. To decide the progession mortality of the disease it was carried out a continuation along six mouths.

Results: The HIV-infeted patients had lower punctuations in all dimensions of SF-36 that control group and population values of reference. The values of Cronbach s alpha coefficient are situated up of 0,7 (range 0,75-0,91) in all measurements. The critical phaseAIDS patients (with AIDS diagnosis and/or $\mathrm{CD}_{4}$ lymphocites $<200 \mathrm{~mm}^{3}$ ) sho wed lower punctuations in all measurements except for mental health. Thirty-seven patients $(14,2 \%)$ displayed new events to AIDS diagnosis and twenty-one died $(8,1 \%)$. The severe immunodepresion (OR: 4,3; CI 95\%: 1,6-11,8), previous AIDS oiagnosis (OR:3,4: CI 95\%: 1,48,1), the physical function dimesion in the SF-36 (OR:0,3; CI 95\%: 0,1-1,1) and the body pain (OR: 0,2; CI 95\%:0,1-0,8) were predictor factors to the progrssion-mortality of the disease.

Conclusions: The SF-36 as a measure of the HRQL in HIV-infected population show a high internal consistence, that is able to discriminate patients with severe immunodepresion and could help to predict more appropriately the progression of the disease.

KEY WORDS: Quality of life. Questionnaires. HIV. AIDS.

García Ordóñez MA, Mansilla Francisco JJ, Nieto Aragón E, Cereto MR, Salas Samper F, Vallejo Díaz, M, Martínez González J. Calidad de vida relacionada con la salud de Pacientes infectados por VIH medida por el Cuestionario de Salud SF-36. An Med Interna (Madrid) 2001; 18: 74-79.

\section{INTRODUCCIÓN}

La infección por el virus de la inmunodeficiencia humana (VIH) y su progresión a síndrome de inmunodeficiencia adquirida (SIDA) origina un deterioro de las funciones físicas, psíquicas y sociales de los pacientes que determina un descenso en la sensación de bienestar. A pesar del considerable esfuerzo realizado en los últimos años en mejorar el tratamiento de la infección por VIH, todavía hay un conocimiento limitado del impacto del tratamiento y de la progresión de la

Trabajo aceptado: 5 de Junio de 2000

Correspondencia: Miguel Ángel García Ordóñez. C/ Horacio Lengo, 17, 4º 29006 Málaga. 
enfermedad sobre la calidad de vida relacionada con la salud (CVRS) (1). Los instrumentos que miden la CVRS, entendida como aquellos aspectos de la vida que se ven afectados directamente por el estado de salud, son cada vez más utilizados para evaluar procedimientos clínicos, intervenciones terapéuticas y calidad de los cuidados suministrados (2). La importancia creciente que ha adquirido la medida de la CVRS en los últimos años refleja su bien establecida validez y el respaldo metodológico que tienen los instrumentos que la miden, así como el interés por conocer la forma en que el paciente percibe y reacciona a su enfermedad (3) Dichos instrumentos pueden clasificarse en dos grandes grupos: genéricos y específicos. Mientras los específicos miden la CVRS en pacientes con una enfermedad concreta, los genéricos pueden ser utilizados en distintos tipos de pacientes o poblaciones con la ventaja de que permiten comparar el impacto de diferentes enfermedades sobre la CVRS.

Cada vez son más utilizados los instrumentos que miden la CVRS en la atención integral de los pacientes infectados por VIH y en los ensayos clínicos que evalúan tratamientos antirretrovirales, ya que detectan cambios en aspectos de la salud que los resultados clínicos tradicionales no reflejan $(4,5)$.

El objetivo del presente estudio fue conocer el impacto de la infección por VIH sobre la CVRS y su relación con variables clínicas e inmunológicas, así como con la progresión y supervivencia de la enfermedad.

\section{MÉTODOS}

Sujetos: Se estudiaron 300 pacientes infectados por VIH en todas las fases de la enfermedad reclutados de la Unidad de Enfermedades Infecciosas del Complejo Hospitalario Carlos Haya de Málaga durante un periodo de 10 meses (1-5-97 al 13-98). Por cada paciente infectado por VIH reclutado se incluyó como control un individuo VIH negativo en seguimiento en nuestra Unidad por hepatopatía crónica viral que presentaba un potencial factor de riesgo para la infección por VIH similar al del caso.

Procedimiento. Una vez obtenido el consentimiento informado, se solicitaba a los participantes que completaran el cuestionario. Los datos sociodemográficos, epidemiológicos y clínicos fueron recogidos de la base de datos de la consulta que es puesta al día en cada visita del paciente. Los participantes fueron seguidos durante un periodo de, al menos, 6 meses con objeto de determinar la aparición de nuevos eventos diagnósticos y la supervivencia durante ese periodo.

Instrumento. Para evaluar la CVRS se ha utilizado el cuestionario de salud MOS SF-36 (36-item Medical Outcomes Study Short-Form General Health Survey). (6-8) El SF-36 es un instrumento genérico, breve y autoadministrado, que contiene 36 items que cubren 8 dimensiones del estado de salud: función física, limitación del rol físico, dolor corporal, percepción de salud general, vitalidad, función social, limitación del rol emocional y salud mental. Detecta tanto estados positivos como negativos de la salud. Los items originan una escala para cada dimensión que tiene un recorrido desde 0 (el peor estado de salud posible para esa dimensión) hasta 100 (el mejor estado de salud) (9). El cuestionario no ha sido diseñado para generar un índice global. Se ha utilizado la recientemente adaptada versión española del SF-36 y sus valores poblacionales de referencia $(10,11)$.
Análisis estadístico. Los datos fueron analizados utilizando los paquetes estadísticos EPIINFO 6.0 y SPSS 6.0.1. Para evaluar la fiabilidad (consistencia interna) se calculó el coeficiente alfa de Cronbach. Para establecer la validez relacionandola con el grado de severidad de la enfermedad se utilizaron marcadores clínicos (estadios del CDC de Atlanta,(12) presencia de eventos diagnósticos definitorios de SIDA) y biológicos (recuento de linfocitos $\mathrm{CD}_{4}$ en sangre periférica). Dado que los valores poblacionales de referencia difieren según el sexo y la edad, y que la gran mayoría de los sujetos del estudio tenían una edad entre 25-34 años, estando el resto de los grupos etarios infrarrepresentados en la muestra, el análisis comparativo de las puntuaciones obtenidas tanto de los casos como de los controles con los valores poblacionales se realizó tomando los individuos comprendidos en la franja etaria de 25-34 años, estratificados por sexo, para hacer equiparable la comparación. Para la comparación de las medias de las puntuaciones obtenidas por el SF-36 de los varones y mujeres en cada subgrupo de edad se utilizó la t de Student. Para conocer la influencia de distintas variables sobre la puntuación del SF36 para cada dimensión se realizó análisis de regresión lineal simple, considerando en cada caso como variable dependiente la puntuación obtenida en cada dimensión. Posteriormente se realizó análisis de regresión lineal múltiple para evitar un posible efecto de confusión de las variables independientes. En el modelo máximo se incluyeron las siguientes variables seleccionadas a partir del análisis de regresión simple: antecedentes de infecciones oportunistas diagnósticas de SIDA, inmunodepresión profunda $\left(\mathrm{CD}_{4}<200\right.$ células $\left./ \mathrm{mm}^{3}\right)$, usuario de drogas intravenosas actual o en el último año, hospitalización en los últimos 3 meses y terapia antirretroviral altamente eficaz (HAART) (13) La estrategia de modelización fue "por eliminación" (backward). Se realizó un diagnóstico de colinealidad utilizando los criterios de Belsey (14). De esta forma se construyó un modelo final para cada dimensión que incluían aquellas variables que influían de forma independiente en la puntuación de las dimensiones del SF-36. La progresión de la infección por VIH, definida por la aparición de nuevos eventos diagnósticos de SIDA, y la supervivencia se evaluaron a los 6 meses de la realización del cuestionario. Para conocer si la CVRS medida por el SF-36 predecía la progresión de la enfermedad y la supervivencia se realizó análisis de regresión múltiple de Cox, llegando a un modelo final por un proceso de eliminación (método backward). El nivel de significación estadística se situó en 0,05. Asimismo, se calcularon los intervalos de confianza al 95\% (IC95\%) de los diferentes estadísticos descriptivos y de comparación.

\section{RESULTADOS}

Los 300 pacientes infectados por VIH incluídos en el estudio tenían una edad media de 32,9 $\pm 6,7$ años y eran varones 229 (76,3\%). Las prácticas de riesgo para la infección VIH incluían: usuarios a drogas intravenosas (202 pacientes; $67,3 \%$ ), transmisión heterosexual (51 pacientes; 17\%), homo/bisexualidad (43 pacientes; 14,3\%) y transmisión por hemoderivados (4 pacientes; $1,3 \%$ ). Noventa y tres pacientes $(31 \%)$ presentaban criterios clínicos de SIDA previo. La cifra media de linfocitos CD4 fue de 276,3 $\pm 225,1$ células $/ \mathrm{mm}^{3}$. Ciento treinta y seis pacientes $(45,3 \%)$ presentaban cifras de $\mathrm{CD}_{4}$ menores de 200 células $/ \mathrm{mm}^{3}, 121$ (40,3\%) entre 200-500 
TABLA I

CARACTERÍSTICAS GENERALES DE LOS PACIENTES

\begin{tabular}{lcc}
\hline & VIH positivos & Controles \\
\hline Sexo (\%) & & \\
Varón & $229(76,3)$ & $222(74)$ \\
M ujer & $71(23,7)$ & $78(26)$ \\
Edad ( \pm DE) & $33,9 \pm 6,7$ & $31,6 \pm 8,6$ \\
Nivel educacional $(\%)$ & & \\
Sin estudios & $35(11,7)$ & $40(13,3)$ \\
Primarios & $168(56)$ & $157(52,3)$ \\
Secundarios & $83(27,7)$ & $92(30,7)$ \\
Superiores & $14(4,6)$ & $11(3,7)$ \\
Prácticas de riesgo (\%) & & \\
UDVP & $202(67,3)$ & $216(72)$ \\
HTSX & $51(17)$ & $48(16)$ \\
HMX & $43(14,3)$ & $36(12)$ \\
Hemoderivados & $4(1,3)$ & - \\
\hline
\end{tabular}

DE: desviación estándar. UDVP: usuarios a drogas vía parenteral. HTSX: transmisión heterosexual. HM X: homosexualidad.

células $/ \mathrm{mm}^{3}$ y $43(14,3 \%)$ superiores a 500 células $/ \mathrm{mm}^{3}$. Los 300 sujetos controles VIH negativos presentaban similares características respecto a edad, sexo, nivel educativo y prácticas de riesgo para la infección por VIH (Tabla I).

Los valores del coeficiente alfa de Cronbach en los pacientes con infección por VIH se situaron entre el 0,75 (función social) y 0,91 (dolor corporal). Valores similares presentaron los controles, tal y como se expresa en la tabla II. En las tablas III y IV se muestran las puntuaciones del SF-36 para cada dimensión de los pacientes infectados por VIH y de los individuos controles, tanto varones como mujeres, para una misma franja etaria (25-34 años). La infección por VIH disminuyó significativamente las puntuaciones de todas las dimensiones en ambos sexos al compararlas con el grupo control y con los valores poblacionales de referencia. Los mayores descensos se obtuvieron en las dimensiones de rol físico, salud general y rol emocional.

Los pacientes en fases avanzadas de la infección por VIH (pacientes con eventos definitorios de SIDA y con recuento de linfocitos $\mathrm{CD}_{4}$ menores a 200 células $/ \mathrm{mm}^{3}$ ) presentaron pun-

TABLA II

COEFICIENTE ALFA DE CRONBACH PARA CADA UNA DE LAS DIM ENSIONES DEL CUESTIONARIO DE SALUD SF-36.

\begin{tabular}{lccc}
\hline \multirow{2}{*}{ Dimensión } & \multirow{2}{*}{ No de items } & \multicolumn{2}{c}{ Alfa de Cronbach } \\
& & Casos & Controles \\
\hline Función física & 10 & 0,91 & 0,92 \\
Rol físico & 4 & 0,90 & 0,86 \\
Dolor corporal & 2 & 0,92 & 0,94 \\
Salud general & 5 & 0,77 & 0,71 \\
Vitalidad & 4 & 0,84 & 0,86 \\
Función social & 2 & 0,71 & 0,72 \\
Rol emocional & 3 & 0,87 & 0,84 \\
Salud mental & 5 & 0,80 & 0,84 \\
\hline
\end{tabular}

TABLA III

PUNTUACIO NES O BTENIDAS PARA LAS DIFERENTES DIM ENSIO NES DEL SF-36 CO RRESPO NDIENTES A PACIENTES INCLUIDOS EN LA FRANJA ETARIA DE 25-34 AÑOS. VARO NES

\begin{tabular}{lccc}
\hline & $\begin{array}{c}\text { VIH positivos } \\
(n=129)\end{array}$ & $\begin{array}{c}\text { Controles } \\
(n=142)\end{array}$ & $\begin{array}{c}\text { Significación } \\
(p)\end{array}$ \\
\hline Función física & $81,9 \pm 22,1$ & $94,1 \pm 12,6$ & $<0,01$ \\
Rol físico & $56,2 \pm 42,5$ & $86,6 \pm 25,9$ & $<0,01$ \\
Dolor corporal & $66,4 \pm 29,8$ & $82,3 \pm 23,2$ & $<0,01$ \\
Salud general & $48,7 \pm 23,6$ & $68,0 \pm 17,4$ & $<0,01$ \\
Vitalidad & $54,3 \pm 25,2$ & $72,5 \pm 14,9$ & $<0,01$ \\
Función social & $69,1 \pm 26,2$ & $80,1 \pm 27,6$ & $<0,05$ \\
Rol emocional & $54,1 \pm 44,4$ & $72,4 \pm 36,1$ & $<0,05$ \\
Salud mental & $60,8 \pm 19,9$ & $72,1 \pm 17,7$ & $<0,05$ \\
\hline
\end{tabular}

aM edia \pm desviación estándar.

\section{TABLA IV}

PUNTUACIO NES O BTENIDAS PARA LAS DIFERENTES

DIM ENSIO NES DEL SF-36 CO RRESPO NDIENTES A PACIENTES INCLUIDOS EN LA FRANJA ETARIA DE 25-34 AÑOS. $M U J E R E S$

\begin{tabular}{lccc}
\hline & $\begin{array}{c}\text { VIH positivos } \\
(n=56)\end{array}$ & $\begin{array}{c}\text { Controles } \\
(n=74)\end{array}$ & $\begin{array}{c}\text { Significación } \\
(p)\end{array}$ \\
\hline Función física & $79,0 \pm 22,9$ & $92,0 \pm 13,6$ & $<0,05$ \\
Rol físico & $59,8 \pm 43,9$ & $83,2 \pm 24,6$ & $<0,01$ \\
Dolor corporal & $61,9 \pm 31,3$ & $80,0 \pm 20,8$ & $<0,05$ \\
Salud general & $41,7 \pm 22,8$ & $73,80 \pm 20,5$ & $<0,01$ \\
Vitalidad & $49,9 \pm 22,8$ & $66,1 \pm 19,5$ & $<0,05$ \\
Función social & $68,1 \pm 28,9$ & $78,6 \pm 25,9$ & $<0,05$ \\
Rol emocional & $51,8 \pm 43,6$ & $69,2 \pm 33,4$ & $<0,05$ \\
Salud mental & $53,7 \pm 23,2$ & $67,2 \pm 19,5$ & $<0,05$ \\
\hline
\end{tabular}

a edia \pm desviación estándar

TABLA V

PUNTUACIONES O BTENIDAS PARA LAS DIFERENTES DIM ENSIO NES DEL SF-36 EN PACIENTES CO N INFECCIÓN POR VIH SEGÚN SITUACIÓN INM UNO LÓ GICA

Dimensión $\quad C D_{4}<200$ células $/ \mathrm{mm}^{3} \quad \mathrm{CD}_{4}<200$ células $/ \mathrm{mm}^{3}$ Significación $<=136$ ) $(n=164)$ (p)

Función física

Rol físico $73,7 \pm 25,5$ $45,1 \pm 44,8$

$83,1 \pm 22,0$ $<0,001$

Dolor corporal $61,8 \pm 30,1$ $68,8 \pm 38,9$ $<0,001$

Salud general $40,1 \pm 24,1$

Vitalidad

Función social $46,3 \pm 25,0$

$69,8 \pm 28,5$ $<0,05$

Rol emocional

$63,1 \pm 28,9$

$48,6 \pm 23,0$ $<0,01$

Salud mental

$44,6 \pm 44,4$

$57,0 \pm 23,1$ $<0,001$

$73,2 \pm 26,2 \quad<0,01$

$59,0 \pm 43,6 \quad<0,01$

aM edia \pm desviación estándar. " no significativo. 
tuaciones significativamente inferiores en todas la dimensiones del SF-36, salvo en salud mental (Tabla V). Por otro lado, los pacientes con antecedentes de drogadicción intravenosa tuvieron puntuaciones menores en las dimensiones de rol físico, dolor corporal, salud general y función social.

Los modelos finales que incluyen las variables influyentes en las puntuaciones de cada dimensión del SF-36 obtenidas por análisis de regresión lineal múltiple se muestran en la tabla VI. La terapia HAART fue la variable que influyó positivamente (mejora de la puntuación) en todas las dimensiones salvo dolor y rol emocional. El antecedente de diagnóstico previo de SIDA disminuyó las puntuaciones de todas las dimensiones a excepción de función social y rol emocional. La inmunodepresión profunda afectó negativamente a todas las dimensiones salvo función física, dolor y salud mental. La hospitalización en lo últimos 3 meses y el antecedente de usuario a drogas intravenosas empeoró las puntuaciones de las dimensiones rol físico, dolor y percepción de salud gene-

\section{TABLA VI}

FACTO RES Q UE DETERM INAN LA PUNTUACIÓ N DEL CUESTIONARIO DE SALUD SF-36 PARA CADA UNA DE LAS DIM ENSIONES EN PACIENTES CON INFECCIÓN POR VIH (ANÁLISIS DE REGRESIÓ N LINEAL M ÚLTIPLE)

\begin{tabular}{|c|c|c|c|c|}
\hline & $\begin{array}{l}\text { Variables } \\
\text { incluidas }\end{array}$ & $\begin{array}{l}\text { Coeficiente } \\
\text { de regresión }\end{array}$ & IC95\% & $\begin{array}{l}\text { Signif. } \\
(p)\end{array}$ \\
\hline \multirow[t]{3}{*}{ Función física } & Constante & 0,80 & 0,$76 ; 0,84$ & 0,001 \\
\hline & SIDA previo & $-0,17$ & $-0,23 ;-0,12$ & 0,001 \\
\hline & Terapia HAART $^{\mathrm{a}}$ & 0,07 & 0,$02 ; 0,13$ & 0,005 \\
\hline \multirow[t]{6}{*}{ Rol físico } & Constante & 0,72 & 0,$62 ; 0.83$ & 0,001 \\
\hline & SIDA previo & $-0,15$ & $-0,27 ;-0,04$ & 0,009 \\
\hline & Terapia HAART & 0,14 & 0,$04 ; 0,23$ & 0,007 \\
\hline & UDVP $^{b}$ & $-0,12$ & $-0,23 ;-0,02$ & 0,018 \\
\hline & Ingreso previo ${ }^{c}$ & $-0,13$ & $-0,30 ; 0,02$ & 0,082 \\
\hline & Inmunodepresión ${ }^{d}$ & d $-0,17$ & $-0,27 ;-0,06$ & 0,002 \\
\hline \multirow[t]{4}{*}{ Dolor corporal } & Constante & 0,80 & 0,$74 ; 0,86$ & 0,001 \\
\hline & SIDA previo & $-0,13$ & $-0,20 ;-0,06$ & 0,001 \\
\hline & UDVP & $-0,13$ & $-0,20 ;-0,06$ & 0,001 \\
\hline & Ingreso previo & $-0,13$ & $-0,24 ;-0,02$ & 0,021 \\
\hline \multirow[t]{5}{*}{ Salud General } & Constante & 0,51 & 0,$44 ; 0,57$ & 0,001 \\
\hline & SIDA previo & $-0,09$ & $-0,15 ;-0,03$ & 0,006 \\
\hline & Terapia HAART & 0,06 & 0,$01 ; 0,12$ & 0,027 \\
\hline & UDVP & $-0,06$ & $-0,12 ;-0,01$ & 0,033 \\
\hline & Inmunodepresión & $-0,05$ & $-0,11 ; 0,01$ & 0,081 \\
\hline \multirow[t]{4}{*}{ Vitalidad } & Constante & 0,54 & 0,$49 ; 0,58$ & 0,001 \\
\hline & SIDA previo & $-0,08$ & $-0,15 ;-0,02$ & 0,017 \\
\hline & Terapia HAART & 0,08 & 0,$03 ; 0,14$ & 0,003 \\
\hline & Inmunodepresión & $-0,08$ & $-0,14 ;-0,02$ & 0,010 \\
\hline \multirow[t]{3}{*}{ Función social } & Constante & 0,68 & 0,$62 ; 0,73$ & 0,001 \\
\hline & Terapia HAART & 0,11 & 0,$04 ; 0,17$ & 0,001 \\
\hline & Inmunodepresión & $-0,12$ & $-0,18 ;-0,05$ & 0,001 \\
\hline \multirow[t]{2}{*}{ Rol emocional } & Constante & 0,59 & 0,$52 ; 0,66$ & 0,001 \\
\hline & Inmunodepresión & $-0,14$ & $-0,25 ;-0,04$ & 0,006 \\
\hline \multirow[t]{2}{*}{ Salud mental } & Constante & 0,57 & 0,$53 ; 0,60$ & 0,001 \\
\hline & Terapia HAART & 0,04 & 0,$02 ; 0,08$ & 0,028 \\
\hline
\end{tabular}

aTerapia antirretroviral altamente eficaz. 'Usuarios a drogas vía intravenosa. 'Ingreso hospitalario en los 3 últimos meses. Inmunodepresión profunda (linfocitos $\mathrm{CD}_{4}$ menores a 200 células $/ \mathrm{mm}^{3}$ ) ral. Las dimensiones que se vieron afectadas por un mayor número de variables fueron rol físico y percepción de salud general.

Treinta y nueve (13\%) de los pacientes con infección por VIH se perdieron durante el seguimiento, por lo que 261 pacientes fueron finalmente evaluables para determinar la progresión y supervivencia de la enfermedad a los 6 meses. Treinta y siete $(14,2 \%)$ pacientes presentaron nuevos eventos diagnósticos de SIDA y $21(8,1 \%)$ fallecieron. Tras análisis de regresión múltiple de Cox, la inmunodepresión profunda (OR: 4,3; IC95\%: 1,6-11,8), la presencia de SIDA previo (OR: 3,4; IC95\%:1,4-8,1) y las puntuaciones obtenidas en las dimensiones función física (OR: 0,3; IC95\%: 0,1-1,1) y dolor corporal (OR: 0,2; IC95\%: 0,1-0,8) del SF-36 formaron parte del modelo final como factores predictores independientes de progresión y mortalidad de la enfermedad.

\section{DISCUSIÓN}

Cada vez son más utilizados los instrumentos que miden la CVRS para evaluar el impacto que las enfermedades crónicas tienen sobre los individuos que las padecen. Así, en los últimos años se ha incrementado el uso de dichos instrumentos en los pacientes con infección por VIH, asociado al desarrollo y evaluación de nuevos tratamientos antirretrovirales, ya que detectan aspectos del estado de salud que los resultados clínicos tradicionales no reflejan $(4,5)$. Estos instrumentos pueden ser utilizados para evaluar cambios de salud en el tiempo, discriminar entre individuos con diferentes estadios de la enfermedad o predecir futuros desenlaces del estado de salud (1) Múltiples instrumentos han sido utilizados para evaluar la CVRS de pacientes con infección por VIH, tanto genéricos (15-20) como específicos (21-24).

En el presente estudio hemos utilizado un instrumento genérico, el MOS SF-36 en su recientemente adaptada versión española (10-11), para evaluar la CVRS en pacientes infectados por VIH. Otras versiones del MOS General Health Survey han sido usadas en dichos pacientes, incluso se han elaborado versiones modificadas específicas para la infección por VIH (1,25-28), habiendo mostrado capacidad para discriminar entre diferentes estadios de la enfermedad y sensibles al cambio en las condiciones clínicas de los pacientes.

La ventaja de utilizar un instrumento genérico de medida de la CVRS reside en la posibilidad de comparar grupos de pacientes con diferentes enfermedades crónicas. De esta forma, las puntuaciones obtenidas en el presente estudio para cada dimensión del SF-36 en los pacientes infectados por $\mathrm{VIH}$, fueron inferiores, no solo a los valores poblacionales de referencia sino también a aquellas obtenidas de un grupo control con una condición crónica subyacente.

Las dimensiones del cuestionario presentaron una alta fiabilidad. Todos los valores del alfa de Cronbach se situaron por encima del 0,7 y en 3 dimensiones los coeficientes fueron iguales o superiores a 0,9 mostrando un alto nivel de precisión (29). Estos valores son comparables a los comunicados en otros estudios que han utilizado otras versiones del MOS en pacientes con infección por VIH (25-27).

El SF-36 fue capaz de discriminar entre pacientes en fases avanzadas de la enfermedad de aquellos en estadios iniciales de la infección por VIH definidas en base a criterios clínicos (estadios de la CDC) y criterios biológicos (recuento de linfo- 
citos $\mathrm{CD}_{4}$ ). Así, los pacientes con criterios definitorios de SIDA y aquellos con inmunodepresión severa (linfocitos $\mathrm{CD}_{4}<200$ células $/ \mathrm{mm}^{3}$ ) presentaron puntuaciones del SF-36 significativamente inferiores en todas las dimensiones salvo en la de salud mental. Esta circunstancia previamente descrita por otros autores (25-27), sugiere que en la evaluación de la CVRS estos instrumentos se deben complementar con la incorporación de medidas de la esfera cognitiva y psicológica. Los pacientes con prácticas de riesgo de drogadicción intravenosa presentaron puntuaciones inferiores en 4 dimensiones (rol físico, dolor corporal, salud general y función social), dato ya apuntado en algunos estudios (26), pudiendo estar en relación con un menor acceso de los mismos a los servicios de salud.

La capacidad de los instrumentos que miden la CVRS para predecir resultados clínicos y el curso de la enfermedad en pacientes con infección por VIH ha sido descrita por diversos autores (30-31). En nuestro estudio, no solo el diagnóstico previo de SIDA y una situación inmunológica muy deteriorada predecían un peor curso clínico, como cabría esperar, sino que además 2 dimensiones del SF-36 (función física y dolor corporal) fueron factores predictores independientes de progresión y mortalidad de la enfermedad. Son precisamente estas dimensiones las que se encuentran más afectadas en estadios avanzados de la infección en otros estudios (25-28). Sin embargo, puede resultar en cierto modo sorprendente que, a pesar de la alta eficacia mostrada por las nuevos tratamientos antirretrovirales (13) la terapia HAART no resultara factor predictivo negativo de progresión de la enfermedad. Posiblemente, seis meses sean un periodo demasiado breve para que muestren su superioridad respecto a otros tratamientos.

Nuestro estudio podría presentar varias limitaciones. En primer lugar, los pacientes provenían de un único centro hospitalario y la muestra se obtuvo en base a la voluntad de participación de los sujetos. A pesar de que la muestra era repre- sentativa en cuanto a prácticas de riesgo y estadios de la enfermedad, los resultados podrían no ser generalizables a la totalidad de la población infectada por VIH. Por otro lado, no se obtuvo medida de los cambios de la CVRS en el tiempo. Una de las aplicaciones de los instrumentos que miden la salud percibida es detectar cambios en la CVRS en un periodo de tiempo determinado. En este sentido, distintas versiones del MOS han mostrado ser sensibles al cambio de la CVRS en el tiempo en pacientes con infección por VIH $(1,24)$. Finalmente, pese a que los instrumentos genéricos tienen la ventaja de que permiten comparar la salud percibida entre grupos de pacientes con diferentes enfermedades, el no haber utilizado un instrumento específico en nuestro estudio ha podido excluir determinados aspectos de la CVRS de particular interés en la población con infección por VIH.

El uso de instrumentos que miden la salud percibida en individuos infectados por VIH es útil ya que permite evaluar el impacto de la enfermedad y elaborar estratégias terapéuticas más beneficiosas. Nuestros resultados sugieren que el cuestionario de salud SF-36 aplicado a pacientes con infección por VIH muestra una alta consistencia interna, es capaz de discriminar a pacientes en fases avanzadas de la enfermedad y podría ayudar a predecir más adecuadamente el curso de la misma.

\section{AGRADECIMIENTOS}

Los autores agradecen a los Drs. Enrique de Ramón Garrido y Juan de Dios Colmenero Castillo el asesoramiento metodológico y la lectura crítica del manuscrito. El cuestionario de salud SF-36 se ha usado con permiso de Medical Outcomes Trust y la versión española adaptada con permiso de Jordi Alonso del Instituto Municipal de Investigación Médica de Barcelona.

\section{Bibliografía}

1. Revicki DA, Wu AW, Murray MI. Change in clinical status, health status, and health utility outcomes in HIV-infected patients. Med Care 1995; 33: AS173-AS182.

2. Guyatt GH, Feeny Dh, Patrick DL. Measuring health-related quality of life. Ann Intern Med 1993; 118: 622-629.

3. Gill TM, Feintein Ar. A critical appraisal of the quality of life measurements. JAMA 1994; 272: 619-626.

4. Franchi D, Wenzel Rp. Measuring health-related quality of life among patients infected with human immunodeficiency virus. Clin Infect Dis 1998; 26: 20-26.

5. Barker S, Tindall B, Carballo M. Quality of life and clinicals trials in HIV infection. Lancet 1990; 335:1045.

6. Ware JE, Sherbourne CD. The MOS 36-item short form health survey (SF-36). I: Conceptual framework and item selection. Med Care 1992; 30: 473-483.

7. McHorney CA, Ware JE, Raczek AE. The MOS 36-item short form health survey (SF-36). II: Psycometric and mental health constructs. Med Care 1993; 31: 247-263.

8. McHorney CA, Ware JE, Lu R, Sherbourne CD. The MOS 36-item short form health survey (SF-36). III: Tests of data quality, scaling assumptions, and reliability across diverse patients groups. Med Care 1994; 32: 40-66.

9. Ware JE, Snow KK, Kosinski M, Gandek B. SF-36 Health Survey. Manual and interpretation guide. Boston MA: The Health Institute, New England, Medical Center, 1993.

10. Alonso J, Prieto L, Antò JM. La versión española del SF-36 Health Survey (cuestionario de salud SF-36): un instrumento para la medida de los resultados clínicos. Med Clin (Barc) 1995; 104: 771-776.

11. Alonso J, Regidor E, Barrio G, Prieto L, Rodríguez C, De la Puente L. Valores poblacionales de referencia de la versión española del cuestionario de salud SF-36. Med Clin (Barc) 1998; 111: 410-416.

12. CDC. 1993 revised certification system for HIV infection and expanded surveillance case definition for AIDS among adolescents and adults. MMWR 1992; 41: 1-19

13. Carpenter CC, Fishl MA, Hammer SM, Hirsh MS, Jacobsen DM, Katzenstein DA, et al. Antiretroviral therapy for HIV infection in 1998: updated recommendations of the International AIDS Society-USA Panel. JAMA 1998; 280: 78-86.

14. Belsey DA. Conditioning diagnostic: collinearity and weak data in regression. New York: John Wiley \& Sons, 1991.

15. Ragsdale D, Morrow JR. Quality of life as a function of HIV classification. Nur Res 1990; 39: 355-359.

16. Willians JBW, Rabkin JG. The concurrent validity of terms in the Quality of Life Index in a cohosrt of HIV-positive and HIV-negative gay men. Controlled Clin Trials 1991; 12: 129S-141S.

17. Gelber RD, Lenderking WR, Cotton DJ, et al. Quality-of-life evaluation in a clinical trial of zidovudine therapy in patients with midly synptomatic HIV infection. Ann Intern Med 1992; 116: 961-966.

18. Lubeck DP, Fries JF. Changes in quality of life among persons with HIV infection. Qual Life Res 1992; 1: 359. 
19. Cleary PD, Fowler FJ, Weissman J, et al. Health-related quality of life in persons with AIDS. Med Care 1993; 31: 569-580.

20. Lenderking WR, Gelber RD, Cotton DJ, et al. Evaluation of the quality of life associated with zidovudine treatment in asynptomatic human inmunodeficiency virus infection. N Engl J Med 1994; 330 : 738-743.

21. Ganz PA, Coscarelli, Schag CA, Kahn B, Petersen L, Hirji K. Describing the health-related quality of life impact of HIV infection: findings from a study using the HIV Overview of Problems-Evaluation System (HOPES). Qual Life Res 1993; 2:109-119.

22. Lubeck DP, Bennet CL, Mazonson PD, Fifer SK, Fries JF. Quality of life and health service use among HIV-infected patients with chronic diarrhea. J Acquir Immune Defic Syndr 1993; 6: 478-484.

23. De Boer JB, Sprangers MA, Aaronson NK, Lange JM, van Dam FS. A study of the reliability, validity and responsiveness of the HIV Overview of Problems Evaluation System (HOPES) in assessing the quality of life of patients with AIDS and synptomatic HIV infection. Qual Life Res 1996; 5: 339-347.

24. Holmes WC, Shea JA. A new HIV/AIDS-targeted quality of life (HAT/QoL) instrument: development, reliability and validity. Med Care 1998; 36: 138-154.

25. Wu AW, Rubin HR, Mathews WC, Ware J, Brysk LT, Hardy WD, et al.
A health status questionnaire using 30 items from Medical Outcomes Study. Med Care 1992; 116: 129-137.

26. Watchel T, Piette J, Mor V, Stein M, Fleishman J, Carpenter C. Quality of life in persons with human immunodeficiency virus infection: Measurement by the Medical Outcomes Study instrument. Ann Intern Med 1992; 116: 129-137.

27. Burguess A, Dayer M, Catalan J, Hawkins D, Gazzard B. The reliability and validity of two HIV-especific health-related quality of life measures: a preliminary analysis. AIDS 1993; 7: 1001-1008.

28. Wu AW, Rubin HR, Mathews WC, et al. Functional status and wellbeing in a placebo-controlled trial of zidovudine in early synptomatic HIV infection. J Acquir Immune Defic Syndr 1993; 6: 452-458.

29. McHorney CA, Tarlov AR. Individual-patient monitoring in clinical practice: are avaible health status surveys adequate?. Qual Life Res 1995; 4: 293-407.

30. Wilson IB, Cleary PD. Clinical predictors of functioning in persons with acquired immunodeficiency syndrome. Med Care 1996; 34: 610-623.

31. Weissman JS, Cleary PD, Seage GR, Gatsonis C, Haas JS, ChasanTaber S, et al. The influence of health-related quality of life and social characteristics on hospital use by patients with AIDS in the Boston Health Study. Med Care 1996; 34: 1037-1056. 